\title{
Shorter Duration of Antibiotics in Low-Risk Febrile Neutropenia in Children with Malignancy
}

\author{
Jagdish Prasad Meena ${ }^{1} \cdot$ Aditya Kumar Gupta ${ }^{1}$ (i) \\ Received: 30 December 2020 / Accepted: 30 December 2020 / Published online: 7 January 2021 \\ (C) Dr. K C Chaudhuri Foundation 2021
}

Febrile neutropenia $(\mathrm{FN})$ is an oncological emergency and one of the most common complications of chemotherapy administration. Prompt evaluation and management of FN are essential for preventing serious morbidity and mortality [1]. The incidence of febrile episodes during chemotherapy is higher with hematological malignancy compared to that of solid tumors, ranging between $10 \%$ to $50 \%$ [2]. The first step in the management of febrile neutropenia after its identification is risk stratification. Risk stratification helps decide the intensity of management of febrile neutropenia. Unlike in adults, there is no universally accepted and well-validated risk-stratification model for pediatric FN. However, the majority of riskstratification methods are based on patient or disease-related risk factors and episode-specific risk factors. Alexander et al. provided a risk stratification model, and it is one of the most commonly used model [3].

Early initiation of antimicrobials and adequate supportive care are the two vital components of pediatric febrileneutropenia management. The choice of empirical antimicrobials is guided by the institutional protocol, which should be based on locally prevailing bacteriological profile and antimicrobial resistance pattern [1]. The choice of in-patient versus out-patient administration of antimicrobials and route of administration is usually dictated by adopting a validated riskstratification strategy, which classifies children into low-risk and high-risk based on clinical and laboratory findings [4]. There are well-established guidelines for the initiation of empirical antibiotics for children presenting with febrile neutropenia. Still, there are no clear-cut guidelines regarding the optimal duration of empirical antibiotics. No recommendations were available to guide the timing of the safe

Aditya Kumar Gupta

adivick@gmail.com

1 Division of Pediatric Oncology, Department of Pediatrics, All India Institute of Medical Sciences, New Delhi 110029, India discontinuation of empirical antibiotics. The majority of available literature recommends discontinuation of empirical antibiotics in patients who have negative blood culture at $48 \mathrm{~h}$ along with evidence of marrow recovery and remain afebrile for at least $24 \mathrm{~h}$. But this remains a piece of low-quality evidence as there is no well-established cut-off for defining marrow recovery. Even though there is some evidence supporting early discontinuation of empirical antibiotics in low-risk febrile neutropenia who have negative blood cultures and who have been afebrile for at least $24 \mathrm{~h}$, irrespective of marrow recovery status, as long as careful follow-up is ensured, the safety of such early discontinuation without marrow recovery is not explored at present [4].

In this issue of the journal, Kumar et al. [5] reported a noninferiority phase- 3 randomized trial of early discontinuation of antimicrobials in pediatric (aged 3-18 y) patients with low-risk FN in an out-patient setting and compared it to standard therapy [continuing antibiotics till recovery of absolute neutrophil counts (ANC) of $\geq 500 / \mathrm{mm}^{3}$ ]. In this study, lowrisk FN patients were started on empirical intravenous antibiotics in an out-patient setting, and randomization was done when the patients became afebrile for at least $24 \mathrm{~h}$. Patients were followed for re-appearance of fever (primary outcome) and re-admission rate (secondary outcome) until ANC recovery. The measure of primary outcome was the proportion of patients remaining afebrile in each arm.

The authors assumed an $85 \%$ success rate in the standard therapy arm and the intervention arm (the stoppage of antibiotics after the patient becomes afebrile) was considered to be noninferior to standard therapy; 71 patients per arm were needed to enroll in this study with an absolute noninferiority margin of $15 \%$. In clinical practice, the noninferiority margin of $15 \%$ looks high. Still, it was calculated based on the authors' center's feasibility trial, and this figure of failure rate is manageable in the low-risk FN patients. A total of 75 patients were randomized to stoppage arm (38) and standard arm (37) in this study over two years. Success rates were $94.6 \%$ in the standard vs. $94.7 \%$ in the stoppage arm. 
The authors should be complimented for this study to report an important unanswered question (optimal duration of antibiotics in low-risk FN). The study's strengths were its randomization nature and enrollment of patients once in the study for each episode of fever to reduce the bias.

However, there are some lacunae that exist in this study. This study could not enroll the desired sample size to make a valid conclusion. With limited power, authors could report a risk difference of $0.1 \%$ [95\% Confidence interval (CI): $-10.0 \%$ to $+10.3 \%$ ] and concluded that the stoppage arm is noninferior to the standard arm.

The two patients in each arm had a failure of treatment. The authors mention that all these patients had profound neutropenia $\left(\mathrm{ANC} \leq 100 / \mathrm{mm}^{3}\right)$. However, it would have been more informative if the authors would have provided the exact ANC value of these patients at baseline before randomization and after having a failed treatment. Readers may be interested to know the diagnosis of patients who had a failure of treatment in this study. The authors enrolled 11 patients of acute myeloid leukemia (AML) in this trial, and even after achieving remission, AML patients receive intensive chemotherapy during consolidation therapy. So it would have been an interesting idea if the ANC values of these patients would have provided. The authors could have planned stratified randomization based on ANC counts and type of malignancy.

The culture positivity was low $(1.2 \%)$ in the study. Multiple exposures to antibiotics for previous febrile episodes during chemotherapy was a reason for low culture positivity explained by authors. It would have been a prudent idea to record the phases of chemotherapy and the number of previous febrile neutropenic episodes at the time of enrollment to know the status of previous exposure to antibiotics.
It can be concluded that there is a need for a randomized study, preferable multicentric with an appropriate sample size with stratified randomization (ANC cutoff, type of malignancy and number of previous febrile neutropenic episodes) to provide an endorsed conclusion for the optimal duration of antibiotics in low-risk febrile neutropenic children.

\section{Compliance with Ethical Standards}

Conflict of Interest None.

\section{References}

1. Oberoi S, Suthar R, Bansal D, Marwaha RK. Febrile neutropenia: outline of management. Indian J Pediatr. 2013;80(2):138-43.

2. Freifeld AG, Bow EJ, Sepkowitz KA, et al. Clinical practice guideline for the use of antimicrobial agents neutropenic patients with cancer: 2010 update by the infectious diseases society of America. Clin Infect Dis. 2011;52(4):e56-93.

3. Alexander SW, Wade KC, Hibberd PL, Parsons SK. Evaluation of risk prediction criteria for episodes of febrile neutropenia in children with cancer. J Pediatr Hematol Oncol. 2002;24(1):38-42.

4. Lehrnbecher T, Robinson P, Fisher B, et al. Guideline for the management of fever and neutropenia in children with cancer and hematopoietic stem-cell transplantation recipients: 2017 update. J Clin Oncol. 2017;35(18):2082-94.

5. Kumar A, Biswas B, Chopra A, Kapil A, Vishnubhatla S, Bakhshi S. Early discontinuation versus continuation of antimicrobial therapy in low risk pediatric cancer patients with febrile neutropenia, before recovery of counts: a randomized controlled trial (DALFEN study). Indian J Pediatr. 2020. https://doi.org/10.1007/s12098-020-03377-x.

Publisher's Note Springer Nature remains neutral with regard to jurisdictional claims in published maps and institutional affiliations. 